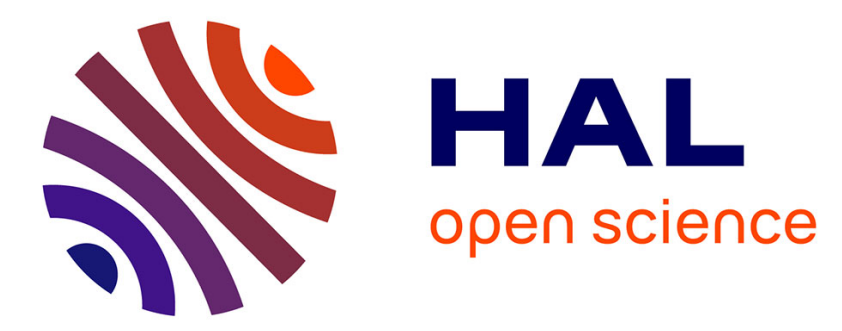

\title{
Van der Waals contribution to the relative stability of aqueous $\mathrm{Zn}(2+)$ coordination states
}

Manoj Ducher, Fabio Pietrucci, Etienne Balan, Guillaume Ferlat, Lorenzo

Paulatto, Marc Blanchard

\section{- To cite this version:}

Manoj Ducher, Fabio Pietrucci, Etienne Balan, Guillaume Ferlat, Lorenzo Paulatto, et al.. Van der Waals contribution to the relative stability of aqueous $\mathrm{Zn}(2+)$ coordination states. Journal of Chemical Theory and Computation, 2017, 10.1021/acs.jctc.7b00455 . hal-01542837

\section{HAL Id: hal-01542837 https://hal.sorbonne-universite.fr/hal-01542837}

Submitted on 20 Jun 2017

HAL is a multi-disciplinary open access archive for the deposit and dissemination of scientific research documents, whether they are published or not. The documents may come from teaching and research institutions in France or abroad, or from public or private research centers.
L'archive ouverte pluridisciplinaire HAL, est destinée au dépôt et à la diffusion de documents scientifiques de niveau recherche, publiés ou non, émanant des établissements d'enseignement et de recherche français ou étrangers, des laboratoires publics ou privés. 


\title{
Van der Waals contribution to the relative
}

\section{stability of aqueous $\mathrm{Zn}(2+)$ coordination states}

\author{
Manoj Ducher, ${ }^{* \dagger}{ }^{\dagger}$ Fabio Pietrucci, ${ }^{\dagger}$ Etienne Balan, ${ }^{\dagger}$ Guillaume Ferlat,${ }^{\dagger}$ Lorenzo \\ Paulatto, ${ }^{\dagger}$ and Marc Blanchard ${ }^{\ddagger}$
}

$\dagger$ †nstitut de Minéralogie, de Physique des Matériaux et de Cosmochimie (IMPMC, Sorbonne Universités, UPMC Univ Paris 06, CNRS UMR 7590, Muséum National

d'Histoire Naturelle, IRD UMR 206, 4 place Jussieu, F-75005 Paris, France

$\ddagger$ GET, CNRS UMR 5563, IRD UR 154, Université Paul-Sabatier, Observatoire

Midi-Pyrénées, 14 avenue Edouard Belin, 31400 Toulouse, France

E-mail: manoj.ducher@impmc.upmc.fr

Phone: +33 1442798 32. Fax: +33 144273785

\begin{abstract}
Many properties of aqueous cations depend on their coordination state. However, the lack of long-range order and the dynamic character of aqueous solutions make it difficult to obtain information beyond average coordination parameters. A thorough understanding of the molecular scale environment of aqueous cations usually requires a combination of experimental and theoretical approaches. In the case of $\mathrm{Zn}^{2+}$, significant discrepancies occur among theoretical investigations based on first-principles molecular-dynamics (FPMD) or free-energy calculations, although experimental data consistently point to a dominant hexaaquo-zinc complex $\left(\mathrm{Zn}\left[\mathrm{H}_{2} \mathrm{O}\right]_{6}\right)^{2+}$ in pure water. In the present study, the aqueous speciation of zinc is theoretically investigated by combining FPMD simulations and free-energy calculations, based on metadynamics


and umbrella-sampling strategies. The simulations are carried out within the density functional theory (DFT) framework using for the exchange-correlation functional either a standard generalised gradient approximation (GGA) or a non-local functional (vdw-DF2) which includes van der Waals interactions. The theoretical environment of $\mathrm{Zn}$ is confronted to experiment by comparing calculated and measured X-ray absorption spectra. It is shown that the inclusion of van der Waals interactions is crucial for the correct modeling of zinc aqueous speciation: whereas GGA incorrectly favours tetraaquo- $\left(\mathrm{Zn}\left[\mathrm{H}_{2} \mathrm{O}\right]_{4}\right)^{2+}$ and pentaaquo-zinc $\left(\mathrm{Zn}\left[\mathrm{H}_{2} \mathrm{O}\right]_{5}\right)^{2+}$ complexes, results obtained with the vdW-DF2 functional show that the hexaaquo-zinc complex is more stable than the tetraaquo and pentaaquo-zinc complexes, by $13 \mathrm{~kJ} \mathrm{~mol}^{-1}$ and by $4 \mathrm{~kJ} \mathrm{~mol}^{-1}$ respectively. These results highlight the critical importance of even subtle interactions for the correct balance of different coordination states in aqueous solutions. However, for a given coordination state, the GGA leads to a reasonable description of the geometry of the aqueous complex.

\section{Introduction}

The reactivity of zinc in aqueous solutions has important consequences in many scientific fields, including biology, geochemistry, or material sciences. Zinc most often occurs in its ionic form $\mathrm{Zn}^{2+}$ with $[\mathrm{Ar}] 3 d^{10} 4 s^{0}$ electron configuration. This filled $d$ shell configuration does not exert a strong control on the coordination state and Zn has been shown to occur in 4-, 5- or 6-fold coordination states in various organic (e.g. proteins) or inorganic phases.

In water, experimental studies point to an average coordination number of 6 at room temperature, with a first solvation shell made of $\mathrm{H}_{2} \mathrm{O}$ molecules only. ${ }^{1-11}$ In comparison, early theoretical studies of the aqueous environment of Zn based on molecular clusters, ${ }^{3,12-16}$ lead to contrasted results, depending on their theoretical level (e.g. MP2 vs. hybrid-DFT) and numerical parameters (e.g. basis quality). ${ }^{13,14,16}$ With increasing computational resources, larger systems were simulated using classical, ${ }^{11,17,18}$ hybrid (QM/MM) ${ }^{19-21}$ and DFT 


\section{Methods}

The calculations presented here are carried out within the DFT framework, ${ }^{25,26}$ using planewaves, periodic boundary conditions and pseudopotentials. Even though DFT is intended to solve exactly the Schrödinger equation in its theoretical conception, its practical implementation requires some approximations. In particular, an exact analytical expression for the electronic exchange-correlation energy is unknown and several approximate formulations exist. The widely used one in condensed matter physics is the Perdew-Burke-Ernzerhof (PBE) parameterisation of generalised gradient approximation (GGA) functional. ${ }^{27}$ By construction, GGA functionals essentially miss van der Waals effects and recent studies emphasised 
on their importance. ${ }^{28-30}$ DFT calculations with non-local functionals are intended to provide a better description of van der Waals interactions through the addition of a non-local correlation term $E_{C}^{n l}$ to the exchange-correlation energy $E_{X C}$ :

$$
E_{X C}=E_{X}^{G G A}+E_{C}^{L D A}+E_{C}^{n l}
$$

where $E_{X}^{G G A}$ is the GGA exchange energy, $E_{C}^{L D A}$ is the LDA correlation energy. Energetic and structural improvements are observed using non-local functionals especially in water. ${ }^{31-34}$ Here we use the vdW-DF2 non-local functional ${ }^{31}$ to evidence improvements over standard GGA calculations in this particular system.

The simulated system contains $1 \mathrm{Zn}$ atom and 64 water molecules in a cubic box of length $12.65 \AA$ so as to reproduce the experimental density of water $\left(1.0 \times 10^{3} \mathrm{~kg} / \mathrm{m}^{3}\right)$. In the initial configuration, the Zn coordination number is 6 . The oxidation state of $\mathrm{Zn}$ being +2 , a negative compensating uniform background charge is applied. All calculations are performed using the PWscf code included in the DFT package Quantum ESPRESSO, ${ }^{35}$ in which Born-Oppenheimer type FPMD is implemented. The time step used, $0.97 \mathrm{fs}$, is chosen to be smaller than the smallest vibrational period in the system (here the $\mathrm{O}-\mathrm{H}$ stretching period of 9-10fs). The simulations are performed in the NVT ensemble, the temperature being maintained at $300 \mathrm{~K}$ using a Berendsen thermostat ${ }^{36}$ with a coupling time constant of 0.3 ps. Ultrasoft pseudopotentials for Zn, O and $\mathrm{H}$ from the GBRV library are used for the description of the ionic cores. ${ }^{37}$ The cut-off energies for wave-functions and charge-density expansions in finite basis-sets are fixed at 50 Ry and 450 Ry respectively. Given the large system size, the Brillouin zone sampling for electronic integration is restricted to the $\Gamma$-point. The instantaneous evolution of $\mathrm{Zn}^{2+}$ coordination is investigated with FPMD trajectories of $30 \mathrm{ps}$ length, amongst which the equilibration and production lengths are 6 and $24 \mathrm{ps}$ respectively.

The thermodynamic properties of the system are then investigated by computing the 
free-energy profiles associated to the changes in Zn coordination with oxygen atoms. These calculations use metadynamics ${ }^{38}$ and umbrella-sampling ${ }^{39}$ strategies which are based on a exploration of the configuration space restricted to the vicinity of a path defined by a collective variable $(\mathrm{CV})$. Here, the $\mathrm{CV}$ is the coordination number $(\mathrm{CN}) s$,

$$
s=\sum_{i=1}^{64} s_{Z n-O_{i}}
$$

where the individual contributions $s_{Z n-O_{i}}$ are calculated using a switching function of the interatomic distances $\left(r_{Z n-O_{i}}\right)$ :

$$
s_{Z n-O_{i}}=\frac{1-\left(\frac{r_{Z n-O_{i}}}{r_{o}}\right)^{n}}{1-\left(\frac{r_{Z n-O_{i}}}{r_{o}}\right)^{m}}
$$

The parameters $r_{0}, n$ and $m$ and were set to $2.8 \AA, 10$ and 20 respectively in order to have a smooth function decaying from 1 to zero between the first and second peak of the Zn-O radial distribution function (see Fig. S1 in the Supporting Information). This choice leads to non-integer numbers for the 3 characteristic coordination numbers (Fig. 1). Nevertheless, in the following, we will describe the coordination states of Zn using $\mathrm{CN}=4, \mathrm{CN}=5$ and $\mathrm{CN}=6$ notations, which correspond to the presence of 4,5 and 6 water molecules within a radius of $2.8 \AA$ from $\mathrm{Zn}^{2+}$ respectively. Metadynamics and umbrella-sampling simulation lengths are 180 ps and 100 ps respectively and are performed using the plugin PLUMED, ${ }^{40}$ which is an open source library for free-energy calculations.

Metadynamics explore the free-energy landscape by depositing a bias potential $V_{B}(s, t)$ in the CV space that facilitates the crossing of free-energy barriers. By doing so, configurations having a low probability of being accessed in FPMD, can be explored in metadynamics in a reasonable time (few ps). The bias potential is obtained by depositing every $60 \mathrm{MD}$ steps, Gaussian hills of height $2.6 \mathrm{~kJ} \mathrm{~mol}^{-1}$ and width of $0.07 \mathrm{CV}$ unit. The profile of the freeenergy $F(s)$ is estimated from the opposite of the final bias potential. The latter is obtained 
by adding the Gaussians deposited during the trajectory at each value of the coordination number, as the time-average of $V_{B}(s, t)$ between the time after which the system has explored all the relevant $\mathrm{CV}$ space $\left(t_{1}=86 \mathrm{ps}\right)$ and the time before which important distortions occur due to the exploration of irrelevant high-energy configurations $\left(t_{2}=138 \mathrm{ps}\right)^{41}$ :

$$
F(s) \simeq-\frac{1}{t_{2}-t_{1}} \int_{t_{1}}^{t_{2}} V_{B}(s, t) d t
$$

By comparing the estimates in the two halves of the interval $\left[t_{1}, t_{2}\right]$, we expect a statistical error bar of $\pm 5 \mathrm{~kJ} \mathrm{~mol}^{-1}$ (Fig. S2 in the Supporting Information).

In umbrella-sampling, instead, the CV space is cut into 20 windows and a harmonic restraining potential of type $k\left(s-s_{c}\right)^{2}$ with a spring constant value $(k)$ of 0.594 Ry is added at the centre $\left(s_{c}\right)$ of each window. $k$ was chosen to give an ideal overlap of 0.9 between consecutive probability distributions. For each window, a trajectory of 5 ps (with the first ps discarded as equilibration) is performed using a configuration extracted from metadynamics as initial structure. The free-energy profile is reconstructed by the weighted histogram analysis method (WHAM). ${ }^{42}$ Free-energy profiles calculated with our in-house WHAM code are similar to those calculated with Grossfield ${ }^{43}$ and GROMACS ${ }^{44}$ codes. By comparing the profiles estimated using only either half of the production trajectory in each window, we expect a statistical error bar of $\pm 2.5 \mathrm{~kJ} \mathrm{~mol}^{-1}$ (Fig. S3 and S4 in the Supporting Information).

Theoretical EXAFS spectra have been computed from the MD configurations using the Feff8 $\operatorname{code}^{45}$ and the methodology detailed in Ref. ${ }^{46}$ Snapshots were extracted every 10 MD steps (after equilibration) from each FPMD trajectory (GGA and vdW-DF2), resulting in two series of about 2,400 configurations. For each configuration, an individual EXAFS spectrum was calculated with SCF potentials within a radius of $3.0 \AA$ around the absorbing atom (Zn), a half-path distance of $7.0 \AA$ and considering all multiple-scattering paths up to 7 legs. The amplitude reduction factor $S_{0}^{2}$ was set to 1 , the value used for the experimental extraction. ${ }^{47}$ An average calculated spectrum $\left(\chi_{\text {calc }}\right)$, directly comparable to the experimental 
one $\left(\chi_{\exp }\right)$, is then obtained from the average over the individual spectra. The agreement between the calculated and experimental spectra is estimated by $\delta$, defined as a sum of squared differences between the $k^{3}$-weighted spectra:

$$
\delta=\sum_{k}\left(k^{3} \chi_{\text {calc }}(k)-k^{3} \chi_{\exp }(k)\right)^{2}
$$

In the following comparisons, two different experimental spectra taken from two recent studies, ${ }^{22,47}$ are used in order to illustrate the error bar associated to the EXAFS signal extraction. These were acquired in diluted solutions of zinc-containing salts $\left(0.1 \mathrm{M} \mathrm{Zn}\left(\mathrm{NO}_{3}\right)_{2}\right.$ in Ref. ${ }^{47}$ and $0.4 \mathrm{M} \mathrm{Zn}\left(\mathrm{ClO}_{4}\right)_{2}$ in Ref. $\left.{ }^{22}\right)$ so that the contribution from counter-ions in the $\mathrm{Zn}$ first neighbours shell can be safely neglected.

\section{Results and discussion}

\subsection{FPMD simulations}

The instantaneous Zn coordination number, calculated using Eq 2 and 3, is shown along both the GGA-PBE and vdW-DF2 trajectories in Fig. 1 (after the 6 ps equilibration time). Representative snapshots of the different Zn complexes observed along the FPMD trajectories are also shown (Fig. 1).

In the GGA-PBE case, after a relatively long duration (12 ps) over which the Zn coordination is close to 6 (hexaaquo complex), it suddenly falls to 5 and then to $\sim 4$ a few picoseconds later. The tetraaquo complex then formed remains stable until the end of the GGA-PBE trajectory, without any return to the $\mathrm{CN}=6$ state (Fig. 1). This suggests that, within the GGA scheme, tetraaquo complexes are more stable than hexaaquo ones and/or the presence of an energy-barrier between $\mathrm{CN}=4$ and $\mathrm{CN}=6$. This is well in line with previous GGA-BLYP simulations (using both long FPMD simulations of 60 ps and free-energy calculations), ${ }^{24}$ which suggested that all 3 complexes co-exist at $300 \mathrm{~K}$, with tetraaquo com- 


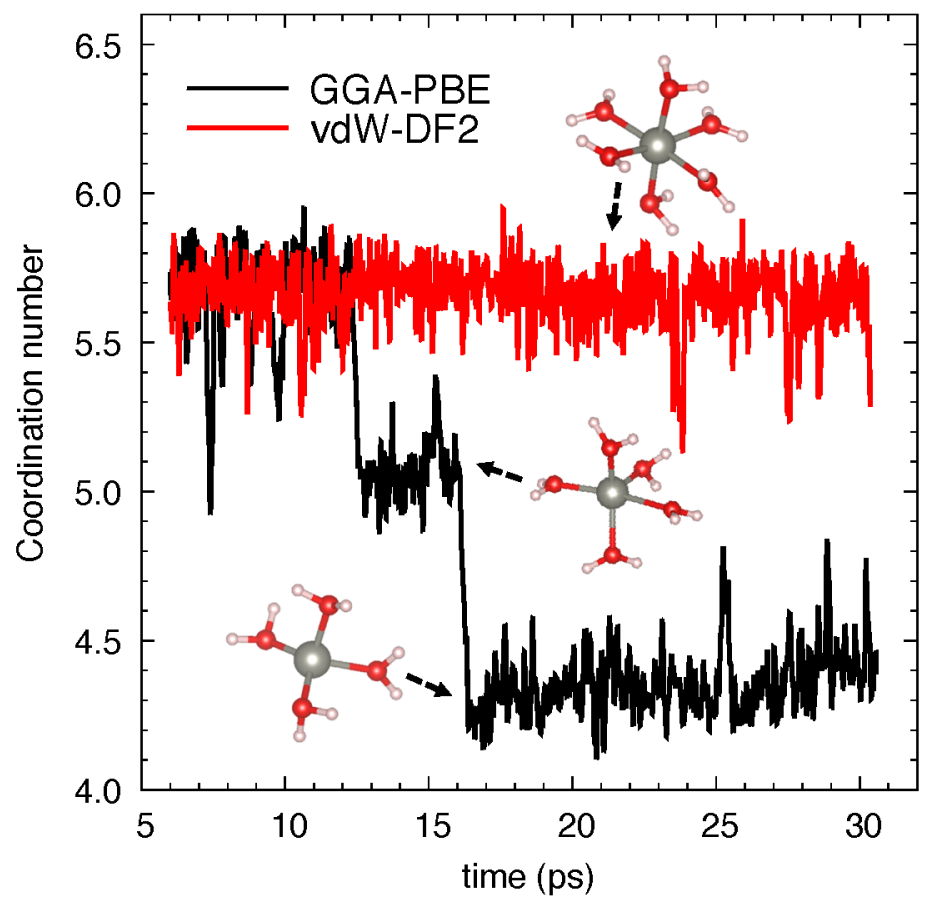

Figure 1 Evolution of the $\mathrm{Zn}$ coordination number in FPMD trajectories performed with GGA-PBE and vdW-DF2 functionals. Representative snapshots of the observed zinc complexes (tetraaquo, pentaaquo and hexaaquo) are shown along the associated trajectories.

plexes more stable than hexaaquo ones by about $11 \mathrm{~kJ} \mathrm{~mol}^{-1}$ (see Fig. 4 later). Although this seemingly contradicts other FPMD simulations (using GGA-PBE) reporting hexaaquo complexes only, ${ }^{22,23}$ one should however note the smaller duration (15 ps) used in these studies: as a matter of fact, this duration is typically comparable to the life-time (12ps) of the (metastable) hexaaquo complex observed in our GGA-PBE trajectory. Thus, the report of $\mathrm{CN}=6$ in these previous studies ${ }^{22,23}$ is likely the consequence of a too short (and incomplete) sampling rather than being the intrinsic (i.e. ergodically sampled) result of the functional used (PBE). This shows the importance of using either long simulation times or enhanced-sampling techniques.

By contrast, in the vdW-DF2 case, Zn is essentially 6-fold coordinated along the whole 30 ps trajectory, except for a few short-lived configurations in which pentaaquo-zinc complexes occur (e.g. between 23 and 24 ps) (Fig. 1). This is indicative of a significant change in the energetics of the system (to be confirmed later, Fig. 5). 

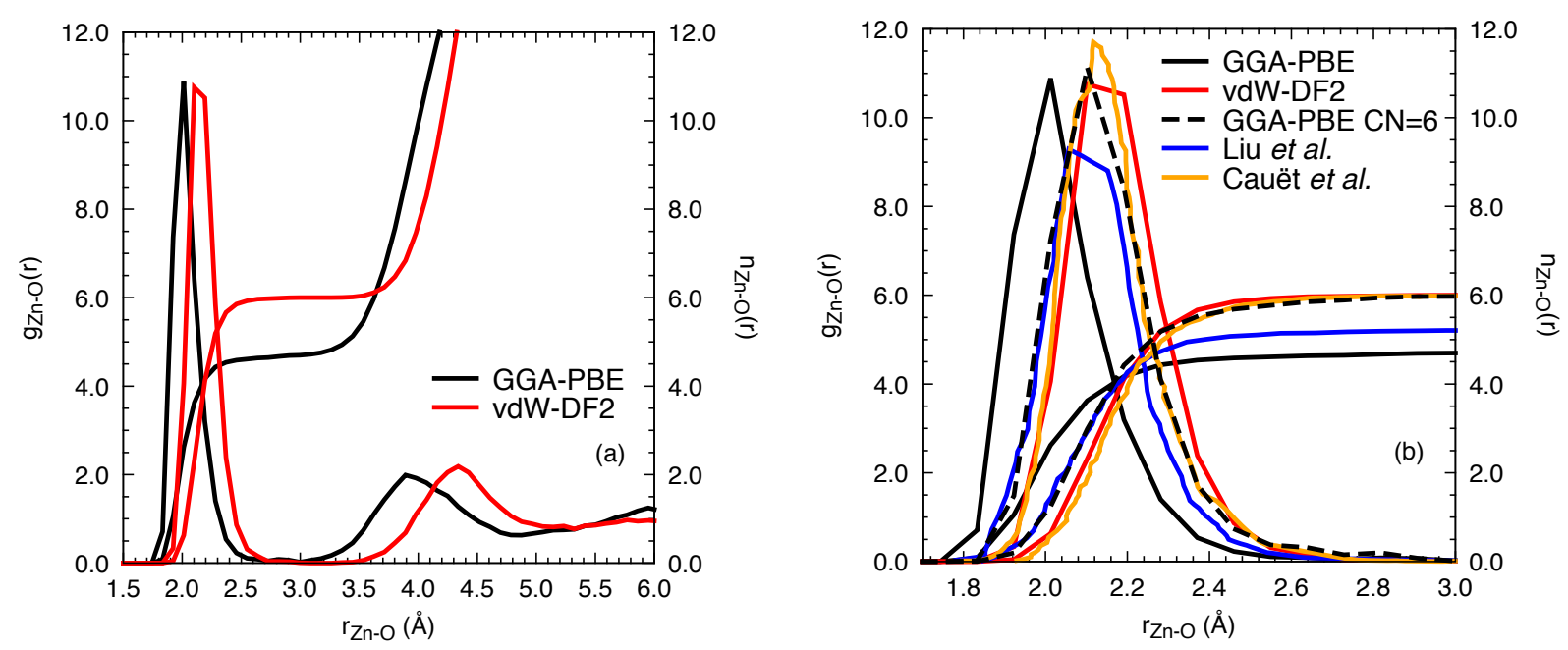

Figure 2 (a) Zinc-oxygen radial distribution function $g_{\mathrm{Zn}-\mathrm{O}}(r)$ and running coordination number $n_{Z n-O}(r)$ obtained from the whole GGA-PBE and vdW-DF2 trajectories. (b) Zoom on the first peak region (1.7-3.0 $\AA$ ). Also added are the results obtained from the portion of the GGA-PBE trajectory where the coordination number is $6(\mathrm{CN}=6)$ and the FPMD (GGA) results from Liu et $a l .{ }^{24}$ and Cauët et al. ${ }^{22}$

The contrasted average Zn speciation between the two trajectories is reflected in the zinc-oxygen radial distribution functions $(\mathrm{RDF}) g_{\mathrm{Zn}-\mathrm{O}}(r)$ and in the corresponding running coordination numbers $n_{\mathrm{Zn}-\mathrm{O}}(r)$ (Fig. 2a). The RDF first peak is located at $r_{\mathrm{Zn}-\mathrm{O}}=2.02$ and $2.14 \AA$ for GGA-PBE and vdW-DF2, respectively. The latter value is within the experimental range of observed Zn-O bond lengths, from $2.06^{9}$ to $2.15 \AA,{ }^{7}$ while the GGA-PBE value is too short. In addition, for the GGA-PBE case, RDF specific to each coordination type $g_{\mathrm{Zn}-\mathrm{O}}^{C N}(r)$ $(\mathrm{CN}=4,5,6)$ have been calculated by using the corresponding portions of trajectory (e.g. from 16 to $30 \mathrm{ps}$ for $\mathrm{CN}=4$, see Fig. 1): the first peak location is $1.97,2.05$ and $2.11 \AA$ for $\mathrm{CN}=4,5$ and 6 , respectively. This shows that the origin of the too short RDF first peak location obtained with GGA-PBE is the overestimation of contributions from the $\mathrm{CN}=4$ and $\mathrm{CN}=5$ complexes. Further, the $g_{\mathrm{Zn}-\mathrm{O}}^{C N=6}(r)$ obtained with GGA-PBE is quite comparable to the vdW-DF2 $g_{\mathrm{Zn}-\mathrm{O}}$ (Fig. $2 \mathrm{~b}$ ): this indicates that although modifying the energetics of the system, the inclusion of van der Waals interactions has only a minor effect on the geometry of the hexaaquo complex. Table 1 summarises structural data extracted from GGA-PBE and vdW-DF2 molecular dynamics trajectories. 
Table 1 Structural information extracted from GGA-PBE and vdW-DF2 FPMD trajectories. The GGA-PBE trajectory is decomposed in three portions as shown in Figure 1 depending on the observed $\mathrm{Zn}$ coordination number $(\mathrm{CN})$ and refered below as $\mathrm{CN}=6, \mathrm{CN}=5$ and $\mathrm{CN}=4$. Information on $\mathrm{Zn}-\mathrm{O}$ radial distribution functions $g_{\mathrm{Zn}-\mathrm{O}}(r)$ as well as the number of water molecules $n_{\mathrm{H}_{2} \mathrm{O}}$, average $\mathrm{Zn}-\mathrm{O}$ distances $r_{\mathrm{Zn}-\mathrm{O}}$ in the first and second solvation shells are reported. Average OZnO angle $<_{\mathrm{OZnO}}, \mathrm{Zn}-\mathrm{H}$ distance $r_{\mathrm{Zn}-\mathrm{H}}, \mathrm{O}-\mathrm{H}$ bond length $r_{\mathrm{O}-\mathrm{H}}$ and $\mathrm{HOH}$ angle $<_{\mathrm{HOH}}$ within the first solvation shell are also given. Root mean square $\sigma$ of these quantities are also reported. Some of these quantities reported by Cauët et al. ${ }^{22}$ are given for comparison.

\begin{tabular}{|c|c|c|c|c|c|c|c|}
\hline & & GGA-PBE & vdW-DF2 & $\begin{array}{l}\text { GGA-PBE } \\
\mathrm{CN}=6\end{array}$ & $\begin{array}{l}\text { GGA-PBE } \\
\mathrm{CN}=5\end{array}$ & $\begin{array}{l}\text { GGA-PBE } \\
\mathrm{CN}=4\end{array}$ & $\operatorname{Ref}^{22}$ \\
\hline \multirow{14}{*}{$\begin{array}{l}1^{\text {st }} \\
\text { solvation } \\
\text { sphere }\end{array}$} & $g_{\mathrm{Zn-O}}(r) 1^{\text {st }} \max$ & 2.02 & 2.14 & 2.11 & 2.05 & 1.97 & 2.12 \\
\hline & $g_{\mathrm{Zn}-\mathrm{O}}(r) 1^{\mathrm{st}} \min$ & 2.8 & 2.8 & 3.0 & 2.6 & 2.35 & 2.8 \\
\hline & $n_{\mathrm{H}_{2} \mathrm{O}}$ & 4.70 & 5.98 & 5.97 & 5 & 4 & 6 \\
\hline & $\sigma_{n_{\mathrm{H}_{2} \mathrm{O}}}$ & 0.84 & 0.14 & 0.16 & & & \\
\hline & $r_{\mathrm{Zn}-\mathrm{O}}$ & 2.13 & 2.19 & 2.17 & 2.10 & 1.99 & 2.18 \\
\hline & $\sigma_{r_{\mathrm{Zn}-\mathrm{O}}}$ & 0.20 & 0.13 & 0.15 & 0.12 & 0.07 & 0.11 \\
\hline & $<\mathrm{OZnO}$ & 98 & 90.1 & 89.9 & 96 & 101 & \\
\hline & $\sigma_{<\mathrm{OZnO}}$ & 12 & 7.7 & 7.9 & 11 & 13 & \\
\hline & $r_{\mathrm{Zn}-\mathrm{H}}$ & 2.67 & 2.78 & 2.77 & 2.67 & 2.58 & 2.76 \\
\hline & $\sigma_{r_{\mathrm{Zn}-\mathrm{H}}}$ & 0.16 & 0.15 & 0.17 & 0.14 & 0.12 & 0.17 \\
\hline & $r_{\mathrm{O}-\mathrm{H}}$ & 1.010 & 0.996 & 1.000 & 1.008 & 1.019 & 0.97 \\
\hline & $\sigma_{r_{\mathrm{O}-\mathrm{H}}}$ & 0.033 & 0.027 & 0.030 & 0.033 & 0.033 & 0.03 \\
\hline & $<_{\mathrm{HOH}}$ & 107.2 & 105.9 & 106.0 & 106.9 & 108.3 & 106.6 \\
\hline & $\sigma_{<\mathrm{HOH}}$ & 5.4 & 5.0 & 5.0 & 5.3 & 5.5 & 5.5 \\
\hline \multirow{6}{*}{$\begin{array}{l}2^{\text {nd }} \\
\text { solvation } \\
\text { sphere }\end{array}$} & $g_{\mathrm{Zn}-\mathrm{O}}(r) 2^{\text {nd }} \max$ & 3.9 & 4.3 & 4.2 & 4.0 & 3.9 & 4.2 \\
\hline & $g_{\mathrm{Zn}-\mathrm{O}}(r) 2^{\text {nd }} \min$ & 4.9 & 5.1 & 4.9 & 4.7 & 4.5 & 4.7 \\
\hline & $n_{\mathrm{H}_{2} \mathrm{O}}$ & 12.2 & 14.7 & 12.0 & 11.84 & 9.82 & 14 \\
\hline & $\sigma_{n_{\mathrm{H}_{2} \mathrm{O}}}$ & 1.2 & 1.5 & 1.1 & 0.93 & 0.92 & \\
\hline & $r_{\mathrm{Zn-O}}$ & 4.12 & 4.37 & 4.33 & 4.10 & 3.95 & 4.22 \\
\hline & $\sigma_{r_{\mathrm{Zn}-\mathrm{O}}}$ & 0.33 & 0.33 & 0.30 & 0.25 & 0.27 & 0.27 \\
\hline
\end{tabular}


We also add in the comparison (Fig. 2b) the results from previous GGA studies. The data from Cauët et al. ${ }^{22}$ (who observed only hexaaquo complexes) are quite similar to our GGA-PBE results restricted to the $\mathrm{CN}=6$ trajectory and to the vdW-DF2 full trajectory (Table 1) while the data from Liu et $a .^{24}$ (who pinpoint the existence of pentaaquo and tetraaquo complexes in however unreported proportions) show a shorter RDF first peak at $2.05 \AA$ (and a running coordination number of 5.2 at $2.8 \AA$ ). This shows that the position of the RDF first peak is a reasonably good marker of the average coordination number, mostly irrespectively of the functional used.

The GGA-PBE and vdw-DF2 scenario are now tested by comparing EXAFS calculated spectra to experimental ones ${ }^{22,47}$ (Fig. 3). The spectrum obtained from the GGA-PBE trajectory shows significant discrepancies with the experimental ones (Fig. 3a): in particular the too large period (in $k$ space) essentially reflects a too short mean Zn-O distance (typically $2.02 \AA$ versus $2.078 \AA$ experimentally ${ }^{47}$ ). A significant improvement is provided with the vdW-DF2 scheme, the residual between the spectra $(\delta)$ being reduced by a factor of about 3-4 (Fig. 3b). A slight frequency misfit is still visible, reflecting in this case a slightly too long mean Zn-O distance. As described previously for the RDF, the GGA-PBE trajectories can be decomposed according to the Zn coordination number (6, 5 and 4), allowing to produce representative spectra for each type of complex (panels c, d and e of Fig. 3). Clearly, the spectrum obtained for hexaaquo complexes (Fig. 3c) shows a pretty good match of the experimental spectra; typically comparable (or slightly better) than that obtained with the vdW-DF2 full trajectory (which corresponds mostly to hexaaquo complexes as well). By contrast, the $\delta$ residual significantly increases for lower coordination types, with a severe frequency misfit most pronounced in the tetraaquo case (Fig. 3e). This decomposition thus allows confirming the main origin of the GGA-PBE deficiency, namely a significant overestimation of contributions from 4-fold and 5-fold complexes. While a quantitative assessment of the relative proportion of each complex from the EXAFS spectra is beyond the scope of the present paper, it seems reasonable to discard the presence of tetraaquo complexes in 

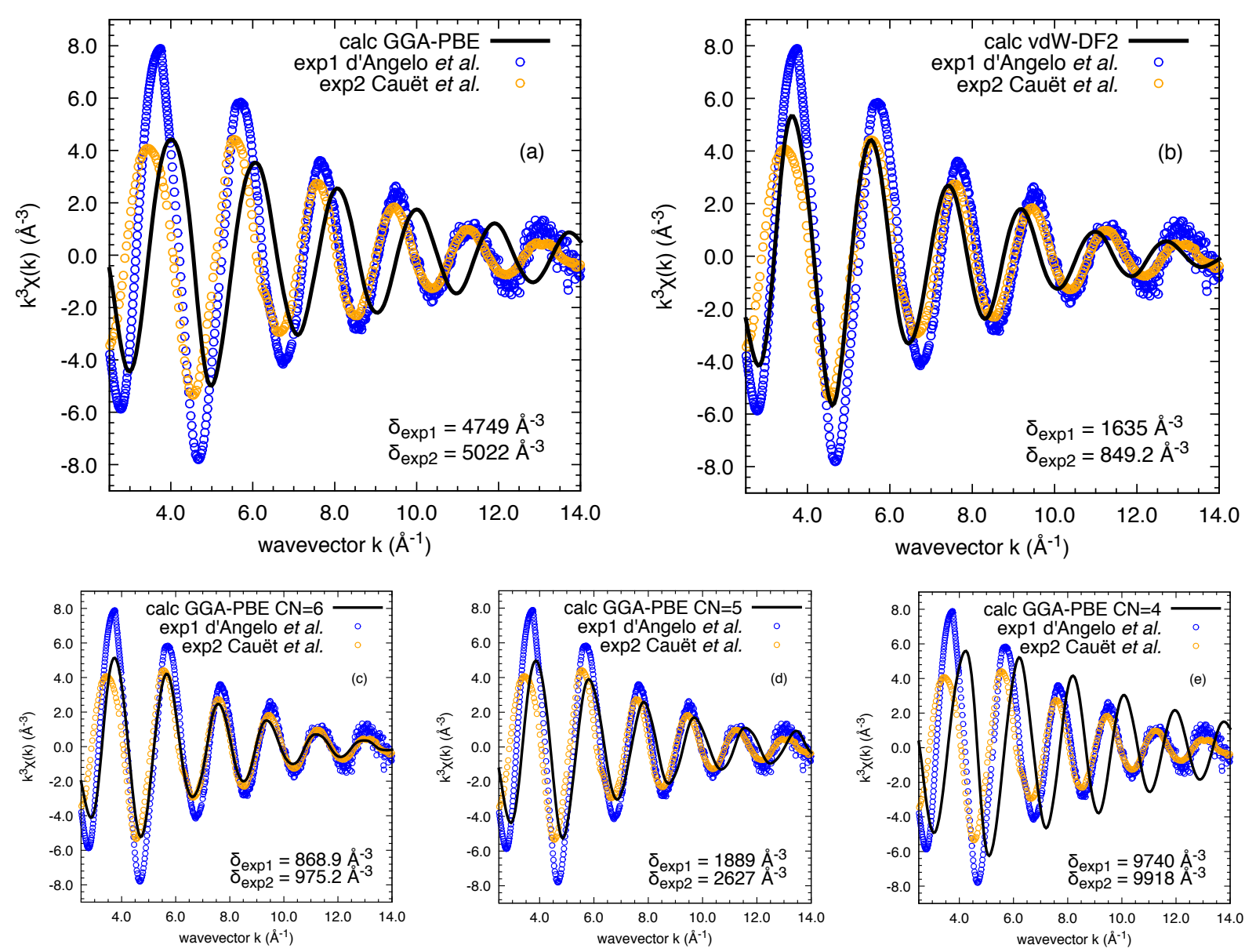

Figure 3 Calculated average EXAFS spectra of molecular configurations extracted from (a) the whole GGA-PBE, (b) the whole vdW-DF2 FPMD trajectories, (c) the portion of GGA-PBE trajectory where $\mathrm{CN}=6$, (d) the portion of GGA-PBE trajectory where $\mathrm{CN}=5$ and (e) the portion of GGA-PBE trajectory where $\mathrm{CN}=4$. These calculated spectra are compared with the experimental spectra from d'Angelo et al. ${ }^{47}$ and Cauët et al. ${ }^{22}$ 
any but marginal proportions. On the other hand, small but possibly sizeable amounts of pentaaquo complexes cannot be ruled out, given that the corresponding EXAFS signal is not very different from the hexaaquo one. In any case, these results highlight serious flaws in the GGA picture obtained both in the present work and in the previous study of Liu et $a l .,{ }^{24}$ which are largely cured within the vdW scheme.

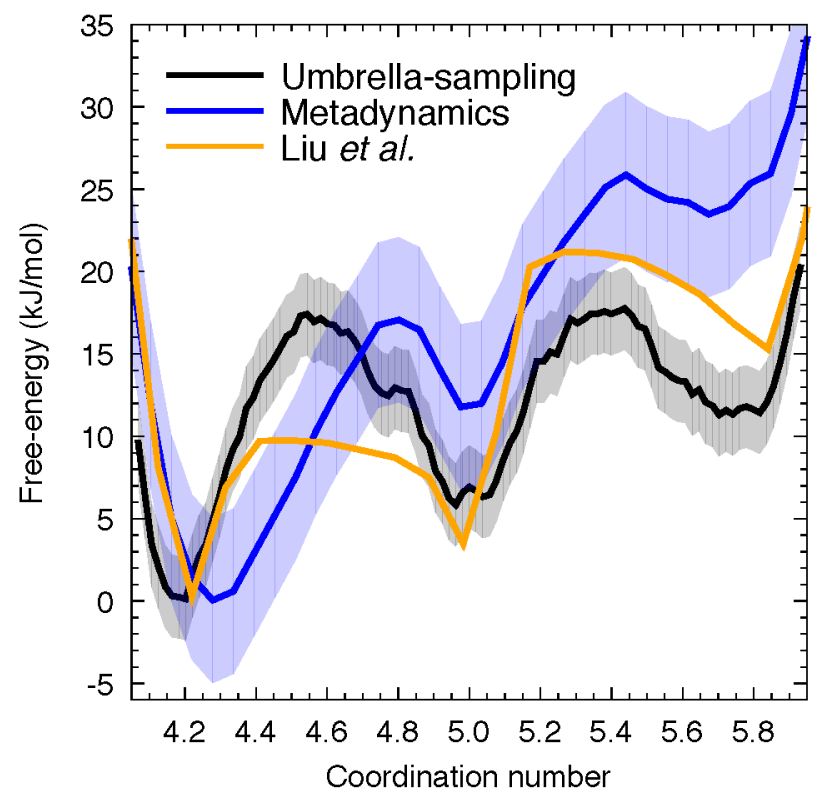

Figure 4 Free-energy profiles of $\mathrm{Zn}$ coordination calculated from metadynamics or umbrellasampling techniques using the GGA-PBE functional. Estimated error bars for each technique are added as shaded areas around the free-energy curves. Results are compared with the freeenergy profile calculated by thermodynamic integration of forces using GGA-BLYP by Liu et al. ${ }^{24}$. Since a slightly different definition of the coordination numbers was used, the values of Ref. ${ }^{24}$ are rescaled to match the values obtained here using Eq 2 and 3.

\subsection{Free-energy profiles}

Free-energy profiles related to changes of $\mathrm{Zn}$ coordination have been determined with the GGA-PBE functional using metadynamics and umbrella-sampling techniques (Fig. 4). Both techniques find hexaaquo complexes to correspond to the highest free-energy minimum of the system followed by pentaaquo and finally tetraaquo. A summary of the free-energy differences and barrier heights between the three complexes are summarized in Table 2. 
These results are consistent with those of Liu et al. ${ }^{24}$ (also reported in Fig. 4 and Table 2), obtained by thermodynamic integration of forces using the GGA-BLYP functional. Beyond the overall good agreement between the techniques, our results show a more pronounced barrier height between $\mathrm{CN}=4$ and $\mathrm{CN}=5\left(17-18 \mathrm{~kJ} \mathrm{~mol}^{-1}\right)$ as compared to those of Liu et al. ${ }^{24}\left(9 \mathrm{~kJ} \mathrm{~mol}^{-1}\right)$, which is likely a result of the different GGA functionals used (PBE versus BLYP). In any case, three distinct techniques used to calculate the free-energy profiles, provide the same overall picture (which is in contradiction with experiments). It shows that within the GGA scheme, unbiased MD simulations starting from equilibrated tetraaquo or pentaaquo complexes have a small probability to sample the hexaaquo state since it requires to overcome a barrier of at least $12 \mathrm{~kJ} \mathrm{~mol}^{-1}$. The reverse situation (sampling $\mathrm{CN}=4$ or 5 starting from $\mathrm{CN}=6$ ) is more likely to happen since the corresponding barriers are lowered to $2-6 \mathrm{~kJ} \mathrm{~mol}^{-1}$. As a matter of fact, GGA FPMD studies with relatively short trajectory lengths $(\sim 15 \mathrm{ps}),{ }^{22,23}$ found only hexaaquo complexes while longer trajectories $(\geq 30 \mathrm{ps})$ display the 3 complexes (this work and Liu et $a l .{ }^{24}$ ).

Table 2 Summary of the free-energy differences $(\Delta F)$ between hexa, penta and tetraaquo-zinc complexes and associated barrier heights calculated from metadynamics using the GGA-PBE functional, and from umbrella-sampling using either the GGA-PBE or the vdW-DF2 functional. The results are compared with those of Liu et al. ${ }^{24}$ obtained from free-energy calculations using the GGA-BLYP functional and thermodynamic integration of forces. Values are given in $\mathrm{kJ} \mathrm{mol}^{-1}$.

\begin{tabular}{lllll}
\hline \hline & $\begin{array}{l}\text { Metadynamics } \\
\text { using GGA-PBE }\end{array}$ & $\begin{array}{l}\text { Umbrella sampling } \\
\text { using GGA-PBE }\end{array}$ & $\begin{array}{l}\text { Umbrella sampling } \\
\text { using vdW-DF2 }\end{array}$ & $\begin{array}{l}\text { Thermodynamic Integration } \\
\text { using GGA-BLYP, from Ref }\end{array}$ \\
\hline$\Delta F_{6-5}$ & 12 & 6 & -4 & 12 \\
$\Delta F_{6-4}$ & 24 & 12 & -13 & 15 \\
$\Delta F_{5-4}$ & 12 & 6 & -9 & 3 \\
barrier $_{6->5}$ & 2 & 6 & 11 & 6 \\
barrier $_{5->6}$ & 14 & 12 & 7 & 18 \\
barrier $_{5->4}$ & 5 & 12 & 14 & 6 \\
barrier $_{4->5}$ & 17 & 18 & 5 & 9 \\
\hline
\end{tabular}

Back to our results in closer details, the free-energy profiles obtained from metadynamics and umbrella sampling agree within $10 \mathrm{~kJ} \mathrm{~mol}^{-1}$ (Fig. 4), as expected from other similar simulations of chemical reactions in solution. ${ }^{48}$ However, two differences regarding the location of the extrema are noticed: first, the coordination number minimum is shifted from 


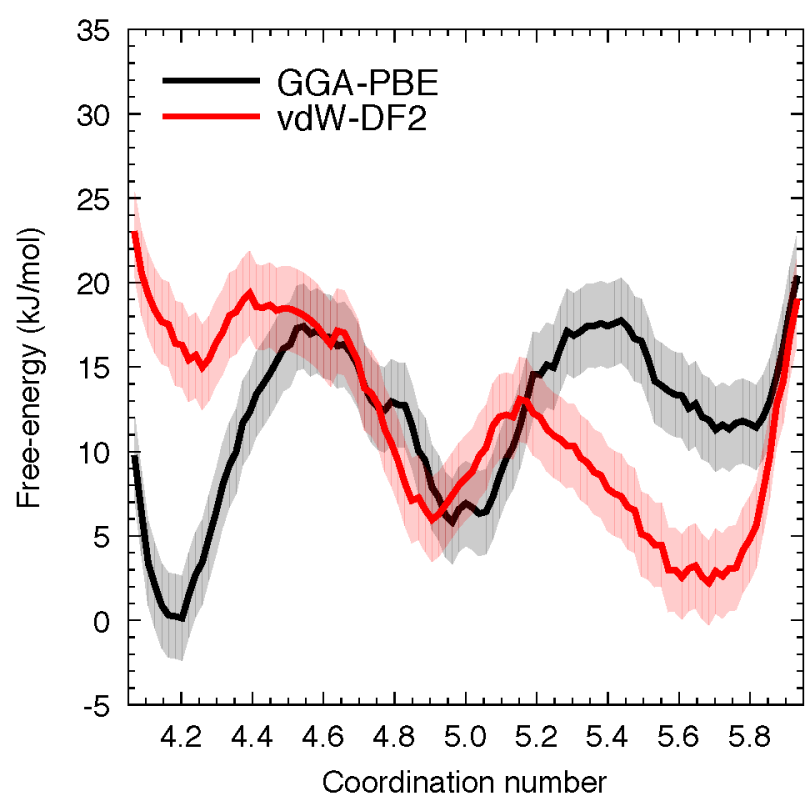

Figure 5 Free-energy profiles of Zn coordination calculated from umbrella-sampling using either the GGA-PBE or the vdW-DF2 functional. Shaded areas correspond to estimated error bars.

We now explore the energetic picture provided by the vdw-DF2 scheme. As the umbrellasampling calculations carried out previously provided a better convergence than metadynamics, ${ }^{49}$ we restricted the study to the former technique only. Remarkably, the free-energy profile calculated with the vdw-DF2 functional gives the very opposite trend relative to that of GGA-PBE (Fig. 5): hexaaquo complexes now correspond to the lowest free-energy of the system followed by pentaaquo and finally by tetraaquo complexes. From the GGA-PBE to the vdW-DF2 description, the energy difference $\Delta F_{6-4}$ between hexaaquo and tetraaquo complexes is reversed from 12 to $-13 \mathrm{~kJ} \mathrm{~mol}^{-1}$. Similarly, $\Delta F_{6-5}$ (the energy difference be- 
tween hexaaquo and pentaaquo complexes) is reversed from 6 to $-4 \mathrm{~kJ} \mathrm{~mol}^{-1}$. The value of the barrier height between $\mathrm{CN}=6$ and $\mathrm{CN}=5$ is $11 \mathrm{~kJ} \mathrm{~mol}^{-1}$, explaining why in vdW-DF2 FPMD trajectory, hexaaquo complexes are mostly observed. Thus the inclusion of vdW forces induces an inversion of the energetic stability of the Zn-aqua complexes which is at the origin of the stabilisation of hexaaquo complexes in FPMD trajectory as evidenced in the previous section (3.1). However, the absolute value of $\left|\Delta F_{6-5}\right|$ is relatively small (as compared to $\left.\left|\Delta F_{6-4}\right|\right)$, thus the presence of a fraction of pentaaquo complexes in aqueous $\mathrm{Zn}$ solutions cannot be ruled out neither from the energetic calculations nor from the EXAFS spectroscopic measurements.

According to the present free-energy calculations, van der Waals interactions are key ingredients to stabilise hexaaquo zinc state with respect to tetraaquo and pentaaquo states at the GGA level. However, in a previous study of aqueous Zn molecular clusters, Diaz et al. ${ }^{16}$ have pointed out that a stabilisation of hexaaquo zinc complex with respect to the tetraaquo one could also be obtained by improving the description of the electronic structure (using calculations at the MP2 level). To further document the respective contribution of electronic exchange and non-local correlation, we have performed additional calculations on the same Zn clusters using hybrid functionals (PBE0 and B3LYP) as well as GGA and vdW functionals (Table S1 in the Supporting Information). On these complexes GGA functionals predict the tetraaquo complex to be the most stable. A better description of the electronic exchange as treated by hybrid functionals leads to the same picture with however a relative stabilising effect on the hexaaquo complex. Only the inclusion of non-local correlation leads to the expected energetic order between the two complexes. Although using a simplified cluster model of Zn environment in water, these additional results point once again towards a dominant role of van der Waals contribution in the relative stability of aqueous $\mathrm{Zn}^{2+}$ coordination states. 


\section{Conclusion}

Using FPMD simulations, free-energy calculations and simulated EXAFS spectra, we have shown that the description of aqueous Zn solutions as obtained from standard GGA functionals suffers from a severe overestimation of the stability of tetraaquo complexes (at the expense of the hexaaquo complexes). Further, the origin of the contrasted results obtained from different FPMD GGA studies ${ }^{22-24}$ has been unveiled. Indeed, because of the presence of large energy barriers, the simulated system can remain kinetically trapped for relatively long times in the (metastable) hexaaquo state: such a situation will fortuitously give a good account of the experimental situation although being numerically unconverged, leaving the problem unnoticed. ${ }^{22,23}$ The incorrect GGA behaviour is revealed by long trajectories and/or enhanced-sampling techniques (here metadynamics and umbrella-sampling) and by a direct confrontation with experiments. The MD-EXAFS combination used here provides a clear enough test of the GGA insufficiencies.

Strikingly, the use of a recent functional including van der Waals contributions, vdw-DF2, changes drastically the relative energetic stability of the aqua complexes, providing a picture fully consistent with experiments in which Zn appears mostly as a hexaaquo complex. This shows that even weak interactions such as the van der Waals forces must be accounted for a reliable description of the chemical and thermodynamical properties of Zn-bearing aqueous solutions. Although the importance of these contributions has been progressively recognised in the recent years in other systems, we are not aware of any other cases in which these forces induce such a marked and qualitative change. From the structural point of view however, it should be noticed that the local geometry of the 6-fold coordination complex is only weakly affected by the use of vdW-DF2 as compared to standard GGA.

The present results may have implications for other hydrated cations, in particular regarding the relative stability of various coordination states. It can be anticipated that the use of non-local functionals incorporating van der Waals contributions could improve the description of main group ions, ${ }^{50,51}$ such as $\mathrm{Ca}, \mathrm{Na}$ and $\mathrm{K}$, which play important biological 
roles or, Cd and Hg. ${ }^{52-57}$

\section{Acknowledgement}

The authors thank P. d'Angelo for providing us with the EXAFS spectra of Ref. ${ }^{47}$ This work was performed using HPC resources from GENCI-IDRIS (Grants 2016-i2016041519 and x2017081875). This work was granted access to the HPC resources of The Institute for scientific Computing and Simulation financed by Region Île de France and the project Equip@Meso (reference ANR-10-EQPX- 29-01) overseen by the French National Research Agency (ANR) as part of the "Investissements d'Avenir" programme. This work was supported by the French national programme EC2CO-Biohefect.

\section{Supporting Information Available}

Calculated GGA-PBE and vdW-DF2 radial distribution functions of Fig 2 along with the switching function defined in Section 2; free-energy profile error bar estimations from metadynamics and umbrella-sampling calculations (PDF); results of electronic exchange and nonlocal correlation contributions to the stability of Zn complexes.

This material is available free of charge via the Internet at http://pubs.acs.org/.

\section{References}

(1) Irish, D. E.; McCarroll, B.; Young, T. F. J. Chem. Phys. 1963, 39, 3436.

(2) Rudolph, W. W.; Brooker, M. H.; Tremaine, P. R. J. Solut. Chem. 1999, 28, 621-630.

(3) Rudolph, W. W.; Pye, C. C. J. Solution Chem. 1999, 28, 1045-1070.

(4) Bol, W.; Gerrits, G. J. a.; van Panthaleon Eck, C. L. J. Appl. Crystallogr. 1970, 3, 486-492. 
(5) Ohtaki, H.; Yamaguchi, T.; Maeda, M. Bull. Chem. Soc. Jpn. 1976, 49, 701-708.

(6) Licheri, G.; Paschina, G. Naturforsch. Tl. A 1982, 37, 1205.

(7) Maeda, M.; T.Ito,; Hori, M.; Johansson, G. Z.Naturforsch. 1996, 51A, 63-70.

(8) Munozpaez, A.; Pappalardo, R. R.; Marcos, E. S. J. Am. Chem. Soc. 1995, 117, 1171011720.

(9) Kuzmin, A.; Obst, S.; Purans, J. Journal of Physics: Condensed Matter 1997, 9, $10065-10078$.

(10) Inada, Y.; Hayashi, H.; Sugimoto, K.-i.; Funahashi, S. J. Phys. Chem. A 1999, 103, $1401-1406$.

(11) D’Angelo, P.; Barone, V.; Chillemi, G.; Sanna, N.; Meyer-klaucke, W.; Pavel, N. V.; Sapienza, L.; Moro, P. a.; Chimica, D.; Federico, N.; Universitario, C.; Via, S. A.; Ii, F.; Angelo, M. S.; Cintia, V. J. Am. Chem. Soc. 2002, 1958-1967.

(12) Mhin, B. J.; Lee, S.; Cho, S. J.; Lee, K.; Kim, K. S. Chem. Phys. Lett. 1992, 197, $77-80$.

(13) Bock, C. W.; Katz, A. K.; Glusker, J. P. J. Am. Chem. Soc. 1995, 117, 3754-3765.

(14) Lee, S.; Kim, J.; Park, J. K.; Kim, K. S. J. Phys. Chem. 1996, 100, 14329-14338.

(15) Pavlov, M.; Siegbahn, P. E. M.; Sandström, M. J. Phys. Chem. A 1998, 102, 219-228.

(16) Díaz, N.; Suárez, D.; Merz Jr, K. M. Chem. Phys. Lett. 2000, 326, 288-292.

(17) Arab, M.; Bougeard, D.; Smirnov, K. Chem. Phys. Lett. 2003, 379, 268-276.

(18) Wu, J. C.; Piquemal, J.-P.; Chaudret, R.; Reinhardt, P.; Ren, P. J. Chem. Theory Comput. 2010, 6, 2059-2070. 
(19) Fatmi, M. Q.; Hofer, T. S.; Randolf, B. R.; Rode, B. M. J. Chem. Phys. 2005, 123, $054514-1$.

(20) Mohammed, A. M.; Loeffler, H. H.; Inada, Y.; Tanada, K. I.; Funahashi, S. J. Mol. Liq. 2005, 119, 55-62.

(21) Brancato, G.; Rega, N.; Barone, V. Chem. Phys. Lett. 2008, 451, 53-57.

(22) Cauët, E.; Bogatko, S.; Weare, J. H.; Fulton, J. L.; Schenter, G. K.; Bylaska, E. J. J. Chem. Phys. 2010, 132, 194502.

(23) Mei, Y.; Sherman, D. M.; Liu, W.; Etschmann, B.; Testemale, D.; Brugger, J. Geochim. Cosmochim. Ac. 2015, 150, 265-284.

(24) Liu, X.; Lu, X.; Wang, R.; Meijer, E. J. Phys. Chem. Chem. Phys. 2011, 13, 1330513309.

(25) Hohenberg, P.; Kohn, W. Phys. Rev. 1964, 136, B864-B871.

(26) Kohn, W.; Sham, L. Phys. Rev. 1965, 385, A1133-A1138.

(27) Perdew, J. P.; Burke, K.; Ernzerhof, M. Phys. Rev. Lett. 1996, 77, 3865-3868.

(28) Langreth, D. C.; Lundqvist, B. I.; Chakarova-Käck, S. D.; Cooper, V. R.; Dion, M.; Hyldgaard, P.; Kelkkanen, a.; Kleis, J.; Kong, L.; Li, S.; Moses, P. G.; Murray, E.; Puzder, a.; Rydberg, H.; Schröder, E.; Thonhauser, T. J. Phys. Condens. Matter 2009, 21, 084203.

(29) Hay, H.; Ferlat, G.; Casula, M.; Seitsonen, A. P.; Mauri, F. Phys. Rev. B - Condens. Matter Mater. Phys. 2015, 92, 1-13.

(30) Gillan, M. J.; Alf, D.; Michaelides, A. J. Chem. Phys. 2016, 144, 130901.

(31) Lee, K.; Murray, É. D.; Kong, L.; Lundqvist, B. I.; Langreth, D. C. Phys. Rev. B Condens. Matter Mater. Phys. 2010, 82, 14. 
(32) Jonchiere, R.; Seitsonen, A. P.; Ferlat, G.; Saitta, A. M.; Vuilleumier, R. J. Chem. Phys. 2011, 135, 154503.

(33) Møgelhøj, A.; Kelkkanen, A. K.; Wikfeldt, K. T.; Schiøtz, J.; Mortensen, J. J.; Pettersson, L. G. M.; Lundqvist, B. I.; Jacobsen, K. W.; Nilsson, A.; Nørskov, J. K. J. Phys. Chem. B 2011, 115, 14149-14160.

(34) Morales, M. A.; Gergely, J. R.; McMinis, J.; McMahon, J. M.; Kim, J.; Ceperley, D. M. J. Chem. Theory Comput. 2014, 10, 2355-2362.

(35) Giannozzi, P.; Baroni, S.; Bonini, N.; Calandra, M.; Car, R.; Cavazzoni, C.; Ceresoli, D.; Chiarotti, G. L.; Cococcioni, M.; Dabo, I.; Dal Corso, A.; de Gironcoli, S.; Fabris, S.; Fratesi, G.; Gebauer, R.; Gerstmann, U.; Gougoussis, C.; Kokalj, A.; Lazzeri, M.; Martin-Samos, L.; Marzari, N.; Mauri, F.; Mazzarello, R.; Paolini, S.; Pasquarello, A.; Paulatto, L.; Sbraccia, C.; Scandolo, S.; Sclauzero, G.; Seitsonen, A. P.; Smogunov, A.; Umari, P.; Wentzcovitch, R. M. J. Phys. Condens. Matter 2009, 21, 395502.

(36) Berendsen, H. J. C.; Postma, J. P. M.; van Gunsteren, W. F.; DiNola, a.; Haak, J. R. J. Chem. Phys. 1984, 81, 3684-3690.

(37) Garrity, K.; Bennett, J.; Rabe, K.; Vanderbilt, D. Comput. Mater. Sci. 2014, 81, 446452.

(38) Laio, A.; Parrinello, M. Proc. Natl. Acad. Sci. U. S. A. 2002, 99, 12562.

(39) Torrie, G. M.; Valleau, J. P. J. Comput. Phys. 1977, 23, 187-199.

(40) Bonomi, M.; Branduardi, D.; Bussi, G.; Camilloni, C.; Provasi, D.; Raiteri, P.; Donadio, D.; Marinelli, F.; Pietrucci, F.; Broglia, R. A.; Parrinello, M. Comput. Phys. Commun. 2009, 180, 1961-1972.

(41) Crespo, Y.; Marinelli, F.; Pietrucci, F.; Laio, A. Phys. Rev. E 2010, 81, 055701. 
(42) Kumar, S.; Rosenberg, J. M.; Bouzida, D.; Swendsen, R. H.; Kollman, P. A. J. Comput. Chem. 1992, 13, 1011-1021.

(43) Grossfield, A. WHAM: the weighted histogram analysis method. version XXX; http: //membrane.urmc.rochester. edu/content/wham.

(44) van der Spoel, D.; Lindahl, E.; Hess, B.; Van Buuren, A.; Apol, E.; Meulenhoff, P.; Tieleman, D.; Sijbers, A.; Feenstra, K.; van Drunen, R.; Berendsen, H. GROMACS user manual version 3.3. 2005.

(45) Ankudinov, A. L.; Ravel, B.; Rehr, J. J.; Conradson, S. D. Phys. Rev. B 1998, 68, 7565.

(46) Ferlat, G.; Soetens, J.-C.; Miguel, A. S.; Bopp, P. A. J. Phys. Condens. Matter 2005, $17, \mathrm{~S} 145$.

(47) D’Angelo, P.; Zitolo, A.; Ceccacci, F.; Caminiti, R.; Aquilanti, G. J. Chem. Phys. 2011, $135,154509$.

(48) Guido, C. A.; Pietrucci, F.; Gallet, G. A.; Andreoni, W. J. Chem. Theory Comput. 2012, 9, 28-32.

(49) Metadynamics simulations required 180 ps of trajectory (from which only a 52 ps window is used to calculate the free-energy profile) while with umbrella-sampling, only 20 trajectories of $5 \mathrm{ps}$ each were used. The latter provided a smaller error bar than metadynamics in a shorter simulation time. Nonetheless, metadynamics was useful to generate reactive trajectories from which we extracted initial configurations for umbrella sampling.

(50) Ohtaki, H.; Radnai, T. Chem. Rev. 1993, 93, 1157-1204.

(51) Marcus, Y. Chem. Rev. 2009, 109, 1346-1370. 
(52) Chillemi, G.; Barone, V.; D’Angelo, P.; Mancini, G.; Persson, I.; Sanna, N. J. Phys. Chem. B 2005, 109, 9186-9193.

(53) Pye, C. C.; Tomney, M. R.; Rudolph, W. W. Can. J. Anal. Sci. Spectrosc. 2006, 51, $140-146$.

(54) D’Angelo, P.; Migliorati, V.; Mancini, G.; Chillemi, G. J. Phys. Chem. A 2008, 112, $11833-11841$.

(55) Kritayakornupong, C.; Plankensteiner, K.; Rode, B. M. Chem. Phys. Lett. 2003, 371, $438-444$.

(56) D’Angelo, P.; Migliorati, V.; Mancini, G.; Barone, V.; Chillemi, G. J. Chem. Phys. 2008, 128, 084502 .

(57) Mancini, G.; Sanna, N.; Barone, V.; Migliorati, V.; D’Angelo, P.; Chillemi, G. Direct 2008, 4694-4702. 


\section{Graphical TOC Entry}

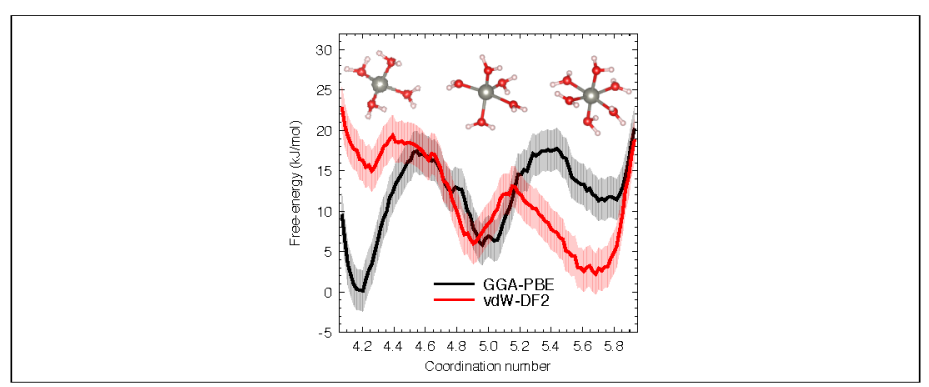

